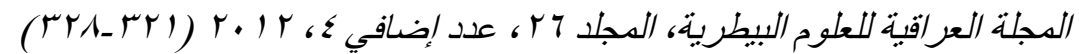

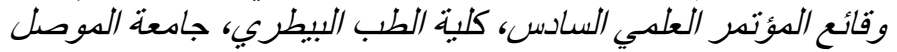

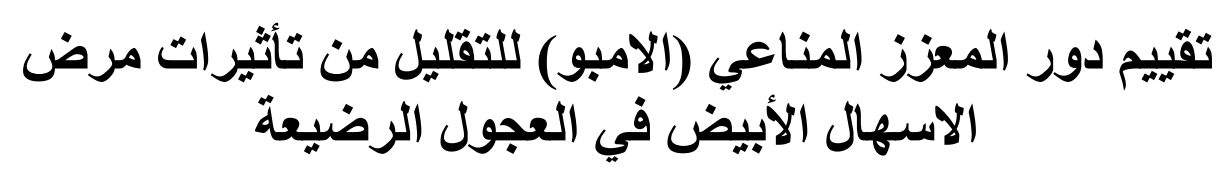

\author{
اسعد جاسب العتابي، جنان محمود خلف و انطوان صبري البنا \\ فرع الطب الباطني و الوقائي، فرع الأحياء المجهرية، كلية الطب البيطري، جامعة بغداد، بغداد، العراق
}

إن الهدف من هذه الدراسة هو لتقييم تأثير استخدام المعزز الحيوي الامبو للتقليل من تأثيرات الإسهال الناتج عن الاشيريشيا القولونية

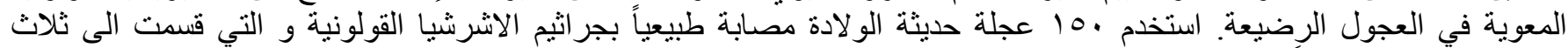

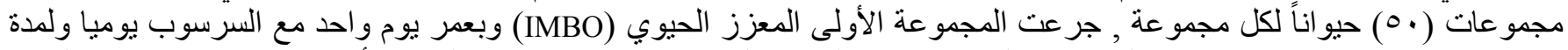

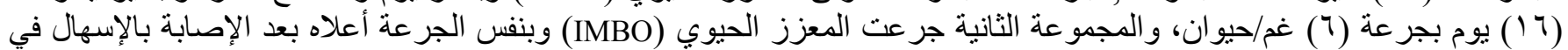

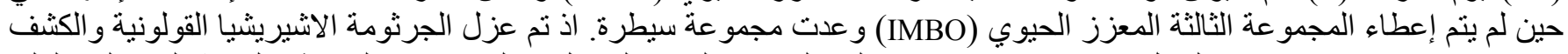

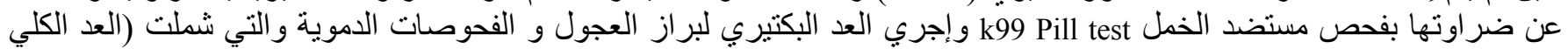

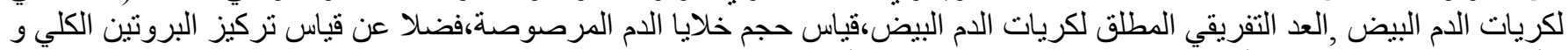

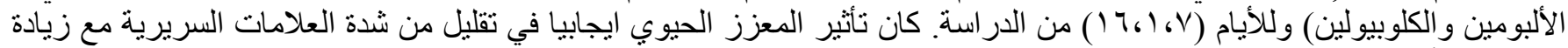

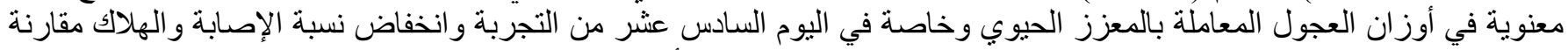

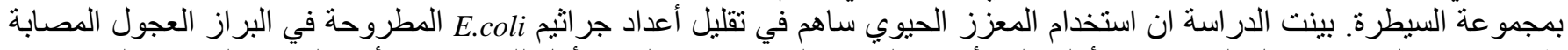

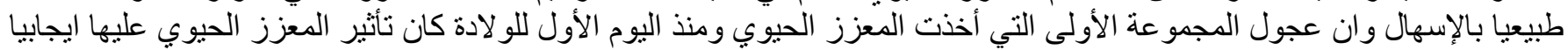

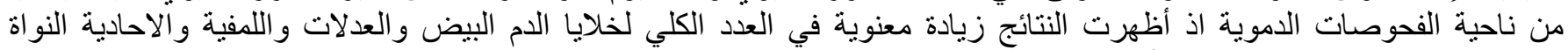

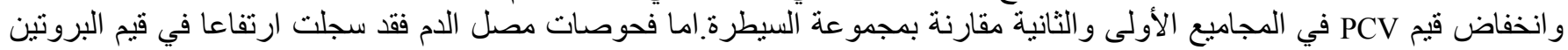

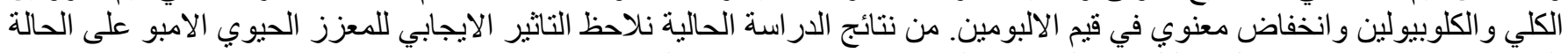
الصحية بعد الإصابة بالإسهال وتقليل شدة العلامات فئل السريرية ورفع الاستجابة المناعية.

\title{
Evaluation of role of probiotic IMBO reduce the effect of collibacilosis in newborn calves
}

\author{
A.J. Aletabi, J.M. Khalaf and A.S. Albana \\ Department of Internal and Preventive Medicine, Department of Microbiology, College of Veterinary Medicine, University of \\ Baghdad, Baghdad, Iraq
}

\section{Abstract}

The aim of this sutdy is to evaluate the effect of using the (IMBO) as probiotic in the reduction of diarrhea caused by E.coli in newborn calves. Using 150 newborn calves naturally infected with E.coli which is divided into three groups 50 animals in each group. First group, the animals of this group were inoculated Biomin IMBO as probioric orally given daily for 16 day with 6 gmlanimal, while second group, the animals of this group were inoculated IMBO as probitic with same previous dose after natural infected with E.coli and suffering from diarrhea. While the third group (control group) which was not inoculated the IMBO as probiotic. The E.coli was isolated and it is virulence was evaluated by $\mathrm{k} 99$ pili test, the bacterial count for the feces sample of the calves was done as many hematological test which include (leukocyte count, differential WBCs count, packed cell volume, as well as TPP concentration and albumin and globulin for day $(1,7,16)$ of the study. The probiotic was positively effective in the decrease of the severity of clinical sings and significantly increase calves weight which were 


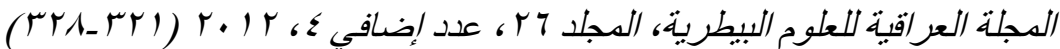 وقائع المؤتدر العلمي السادم، كلية الطب البيطري، جامعة الدوصل}

treated by the probiotic, in the day (16) of the study and increase in the mortality rate compared with control group. The study shows probiotics use contributed in decrease of the number of E.coli excreted by feces of naturally infected calves with colibacillosis. Hematology test results showed significant increase in total leukocyte count, neutrophil, monocyte count and significant decrease in pact cell volume in the first and second group compared with control group. While serum test showed significant increase in total protein and globulin and significant decrease in albumin value. From the results of the study we notice the positive effect of probiotic IMBO on the health state after causing infection by colibacillosis and decrease the severity of clinical signs and increase the immunity respond.

Available online at http://www.vetmedmosul.org/ijvs

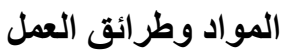

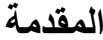

اجريت الدراسة على ،10 عجلة حديثة الولادة من سلالة

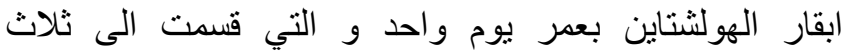

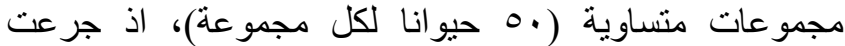

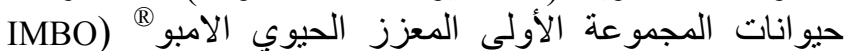

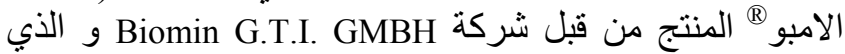
يحتوي على جرثومة Enterococcus Faecium

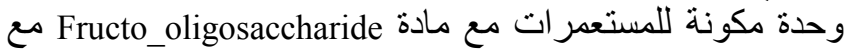

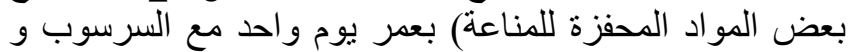

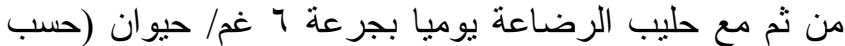

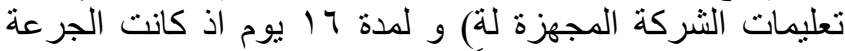

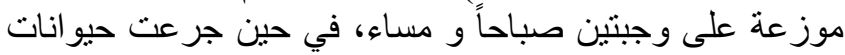

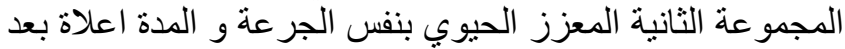

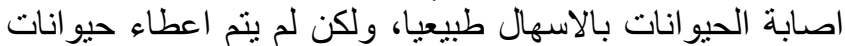

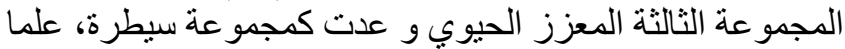

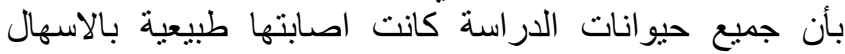

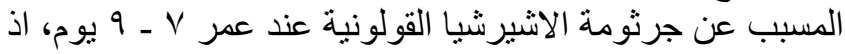

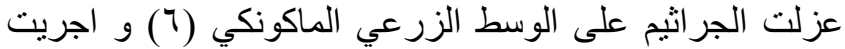

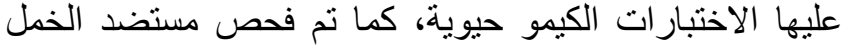

(V) K99 pill test كما تم وزن كل الحويانات و اجراء الفحص السريري علئ العيها

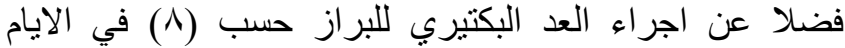

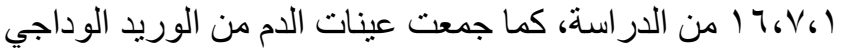

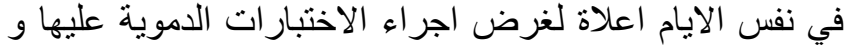

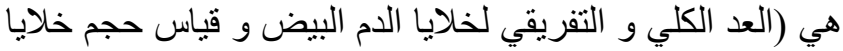

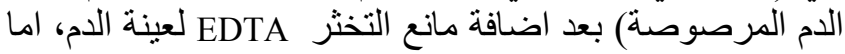

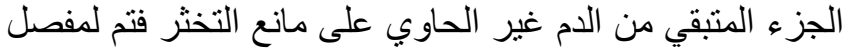

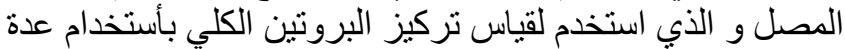
قياس جاهزة و المنتج من قبل شركة Spinoreact.S.A الاسبانية و بواسطة جهاز المطياف الضونئي فئي الابطالي. حللت النتائج إحصائيا باستخدام البرنامج الإحصائي SPSS، الإئي وقدر الوسط الحسابي والخطا القياسي، وحلات البيانات باستخدام الإئي
هنالك العديد من العوامل المسببة للإسهال ومن أهمها

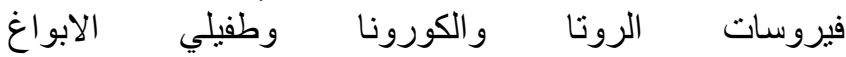

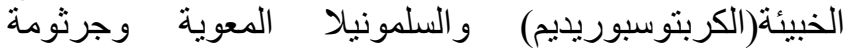

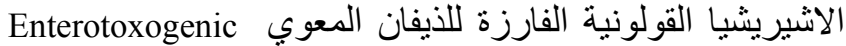
E.coli

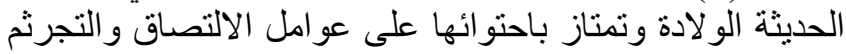

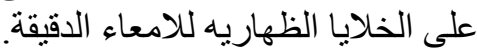

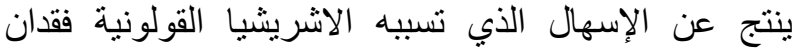

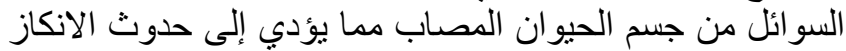

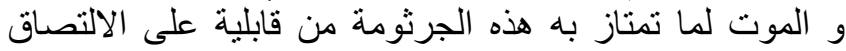

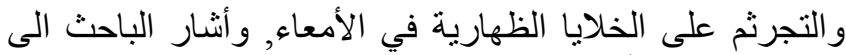

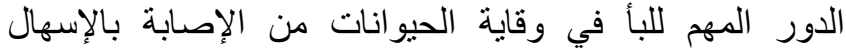
الأبيض في العجول لاحتوائه مقادير كبيرة من الأجسام المضادة الأبات

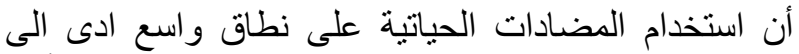

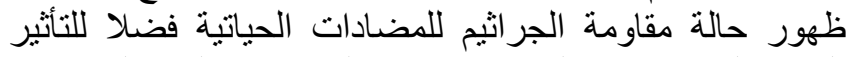

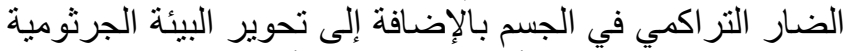

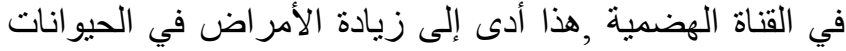

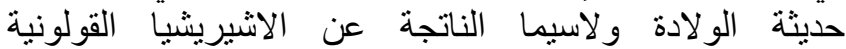

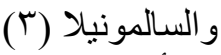

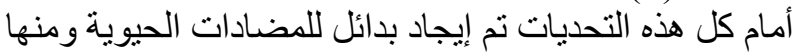
المعززات الحيوية والتي تعرف على التى انها جرانيات اثليم غير مرضيه

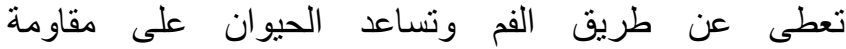
الإمر اض,وكذللك لها تأثير كبير في تحسين معدلات النير النمو والحد

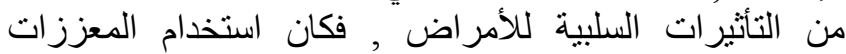

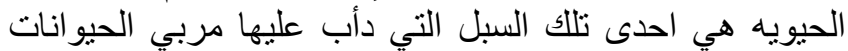

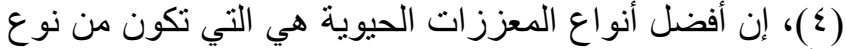

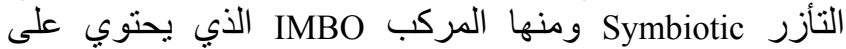

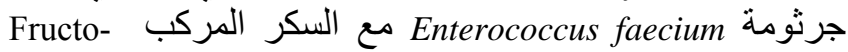
سكر الفركتوز الاحادي يكون بشكل سلاسل فيكل فيكاة قصيرة من السكريات تشجع نمو البكتيريا النافعة في القناة

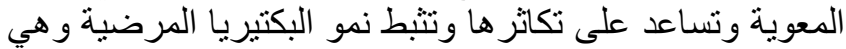
لاتهضم بو اسطة انزيمات المضيف (0) (0). 
الخلايا الأحادية Monocytes و البلعميات اذ تؤثر بصورة غير

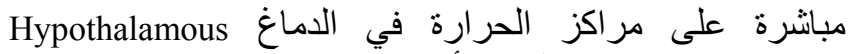

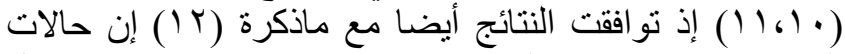

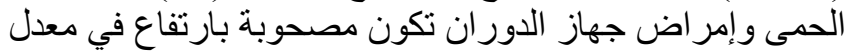

التنفس.

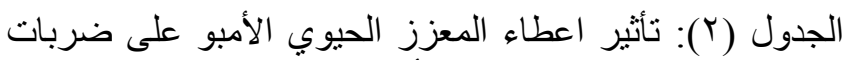

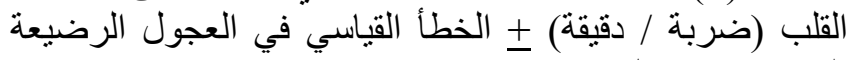

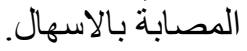

\begin{tabular}{|c|c|c|c|}
\hline \multicolumn{3}{|c|}{ المجاميع } & \multirow[b]{2}{*}{ الايام } \\
\hline (مجمو عة & الثانية (معزز حيوي & حيوي من الاولى (معزز & \\
\hline $107 \pm 127$ & $1.3 \pm 122$ & $2.1 \pm 125$ & 1 \\
\hline$\underset{\mathrm{a}}{2.8+161}$ & $2.6+\frac{1}{b} 137$ & $2.0+\frac{+135}{b}$ & 7 \\
\hline $\begin{array}{c}2.6 \pm 131 \\
\mathrm{a}\end{array}$ & $\begin{array}{c}1.8 \pm 124 \\
\mathrm{a}\end{array}$ & $\begin{array}{c}2.3 \pm 126 \\
\mathrm{a}\end{array}$ & 16 \\
\hline
\end{tabular}

الحروف المختلفة أفقيا تشير اللى وجود فرق معنوي عند مستوى معنوية (P<0.05).

الجدول (ب): تأثثير اعطاء المعزز الحيوي الامبو على التنفس

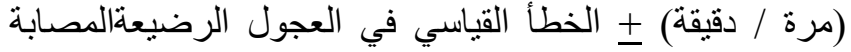

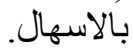

\begin{tabular}{|c|c|c|c|}
\hline \multicolumn{3}{|c|}{ المجاميع } & \multirow[b]{2}{*}{ الايام } \\
\hline (مجيطو عة & الثانية (معزز حيوي & حيوي من الاولى (معزز & \\
\hline $\begin{array}{c}0.4+14.0 \\
\mathrm{a}\end{array}$ & $0.3+13.0$ & $\begin{array}{c}0.4+14.0 \\
\mathrm{a}\end{array}$ & 1 \\
\hline$\underset{\mathrm{a}}{1.2 \pm 29.0}$ & $\frac{0.7 \pm 1}{b}$ & $\frac{0.9 \pm 17.0}{b}$ & 7 \\
\hline $\begin{array}{c}1.0+17.0 \\
\text { (a) }\end{array}$ & $\begin{array}{c}0.3 \pm 14.0 \\
(\mathrm{~b})\end{array}$ & $\begin{array}{c}0.6+15.0 \\
(\mathrm{~b})\end{array}$ & 16 \\
\hline
\end{tabular}

الحروف المختلفة أفقيا تشير الى وجود فرق معنوي عند مستوى (a)05 معنوية (P<0.05).

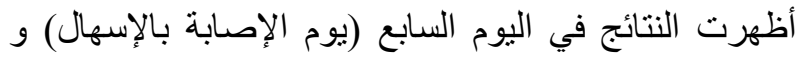

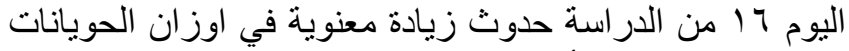

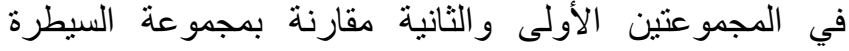

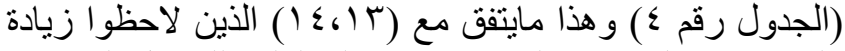

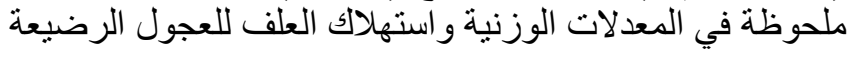

التحليل البياني One away analysis of varaince وقد تم تحديد الاختلافات الإحصائية بين المجاميع المختلفة باستخدام اختبار

(9) Duncan

النتائج والمناقشة

بلغت نسبة الإصابة بالاسهال لحيو انات مجمو عة السيطرة \% اذ لوحظ عليها علامات الخمول وفقدان الثهية وتدهور الإنهات حالتها

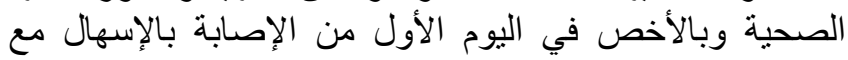

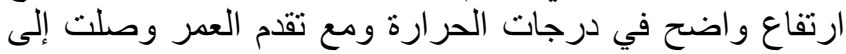

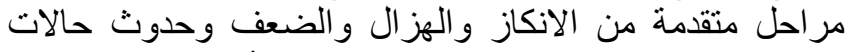

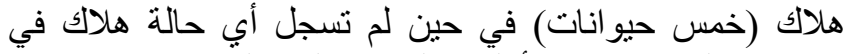

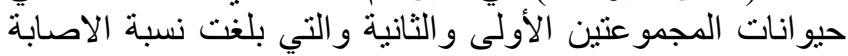

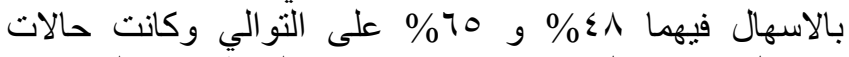

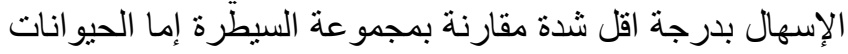
التي لم تهلك في مجمو عة السبطرة فقد بقيت هزيلة ولته وضعيفة. أظهرت نتائج الدراسة السريرية ارتفاعا معنويا في معدلات

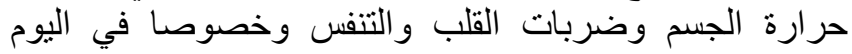
السابع (معدل يوم الإصابة الطبيعية بالإسهال) وكما موضح في في التي

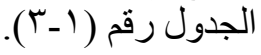

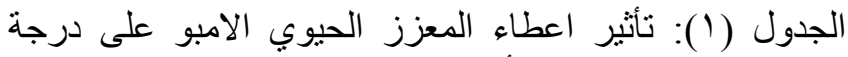

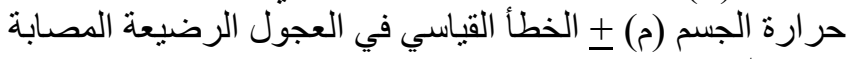

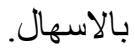

\begin{tabular}{|c|c|c|c|}
\hline \multicolumn{3}{|c|}{ المجاميع } & \multirow[b]{2}{*}{ الايام } \\
\hline (السبطرة عة & 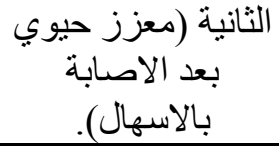 & حيوي من الاولى (معزز & \\
\hline $\begin{array}{c}0.06 \pm 38.9 \\
\mathrm{a}\end{array}$ & $\begin{array}{c}0.04 \pm 38.8 \\
\mathrm{a}\end{array}$ & $0.06 \pm 38.9$ & 1 \\
\hline$\frac{0.07 \pm 40.0}{\mathrm{a}}$ & $\frac{0.06+39.4}{\mathrm{~b}}$ & $\frac{0.06+39.4}{\mathrm{~b}}$ & 7 \\
\hline $\begin{array}{c}0.09 \pm 39.0 \\
\mathrm{a}\end{array}$ & $\begin{array}{c}0.07 \pm 38.9 \\
\mathrm{a}\end{array}$ & $\begin{array}{c}0.05 \pm 38.9 \\
\mathrm{a}\end{array}$ & 16 \\
\hline
\end{tabular}

و هذا الارتفاع في تللك المعايير ناتجا عن دخول جراثيم

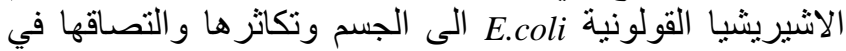

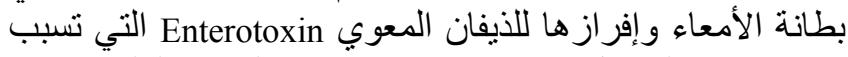

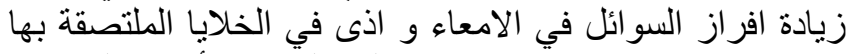

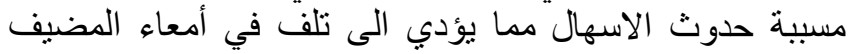

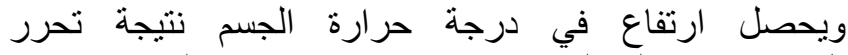

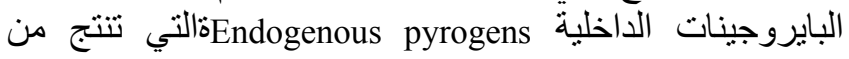


المعززات الحيوية تقلل من أعداد جراثيم المطروحة في براز العجول بعد اصنابتها تجريبيا (الجدول آج). الجدول (ج): تأثير اعطاء المعزز الحيوي على العد البكتيري

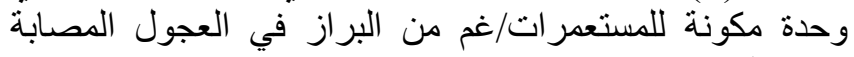

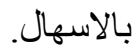

\begin{tabular}{|c|c|c|c|}
\hline \multicolumn{3}{|c|}{ المجاميع } & \multirow[b]{2}{*}{ الايام } \\
\hline (مجيطو عة & الثانية (معزز حيوي & حيوي من الاولى (معزز & \\
\hline $\begin{array}{c}1.4 \pm 1.1 \times 10^{8} \\
\mathrm{a}\end{array}$ & $\begin{array}{c}9.2 \pm 1.9 \times 10^{7} \\
b\end{array}$ & $\begin{array}{c}8.7 \pm 1.4 \times 10^{4} \\
b\end{array}$ & 7 \\
\hline$\underset{\mathrm{a}}{1.2 \pm 2.4 \times 10^{8}}$ & $\begin{array}{c}0.3 \pm 1.7 \times 10^{5} \\
b\end{array}$ & $0.09 \pm \frac{1}{b} \times 10^{2}$ & 16 \\
\hline
\end{tabular}

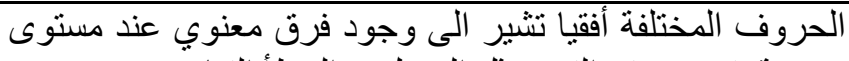

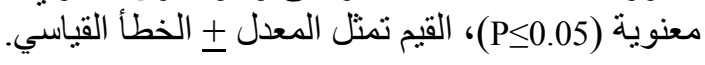

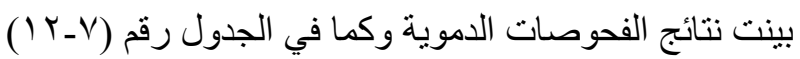

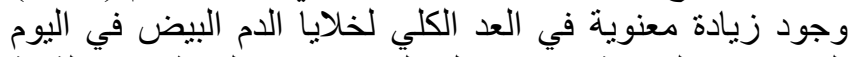

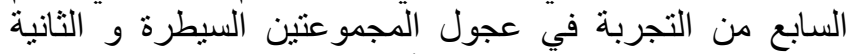

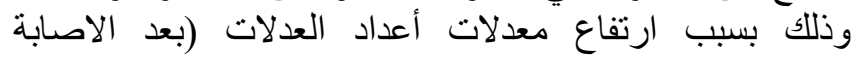

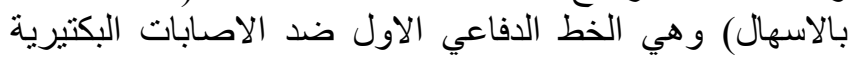

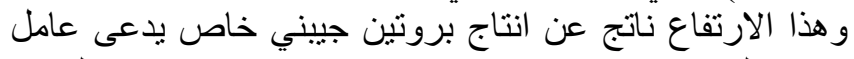

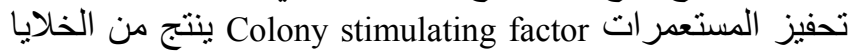
البلعمية كاستجابة للاصنابات البكتيرية (1) (1).

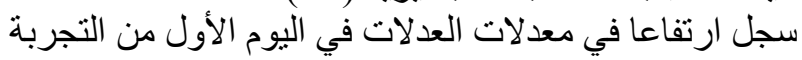

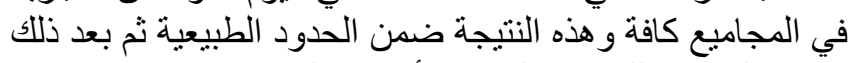

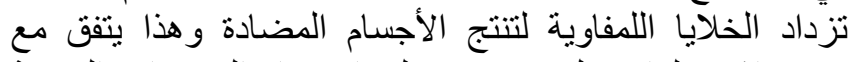

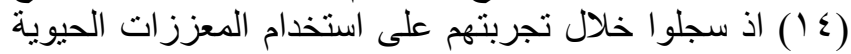

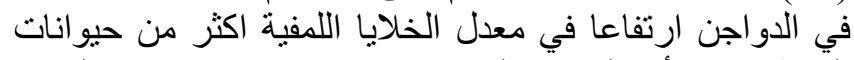

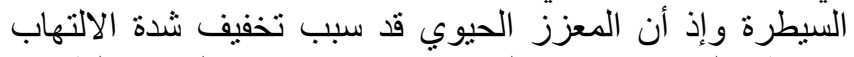

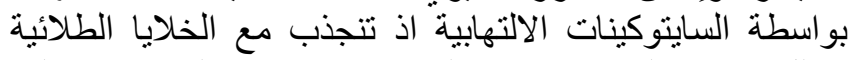

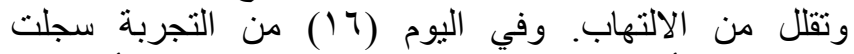

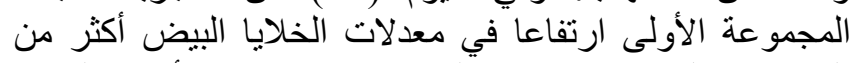

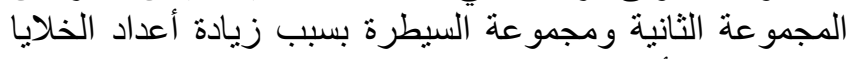

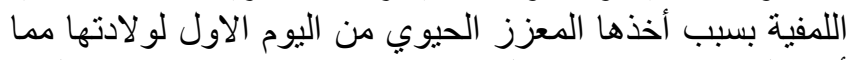

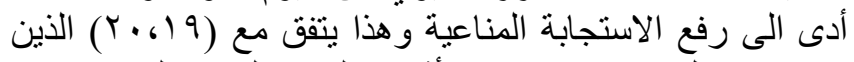

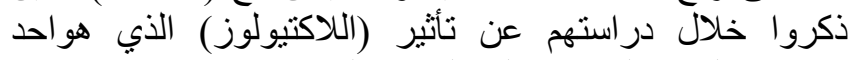

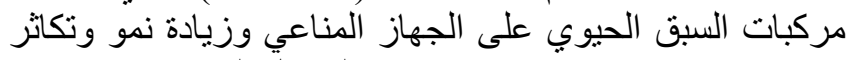

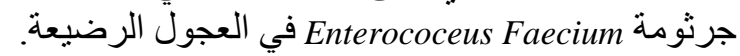

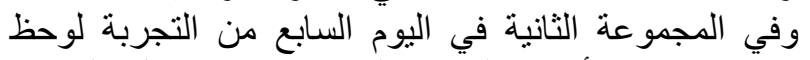

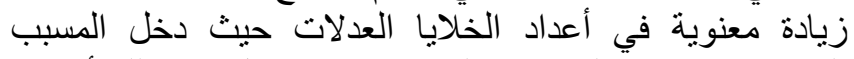

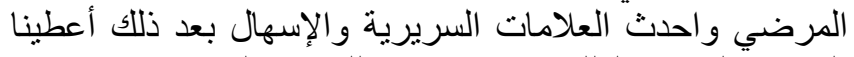

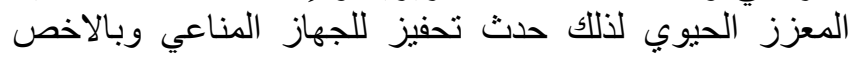

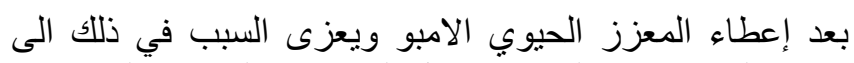

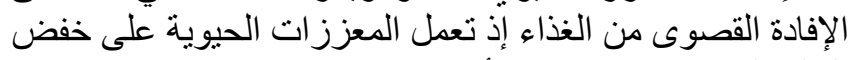

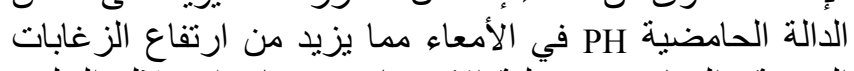

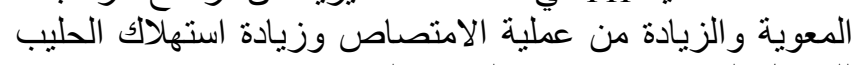

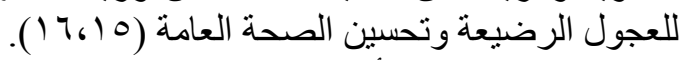

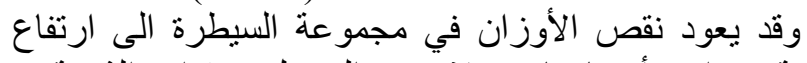

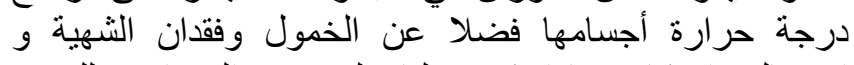

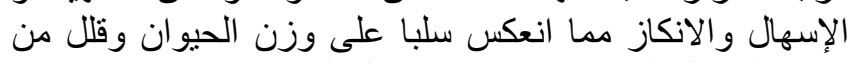

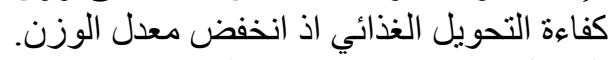

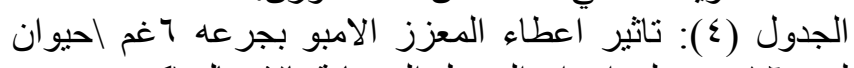

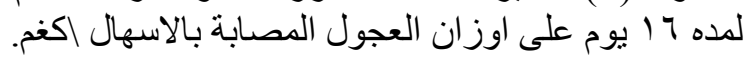

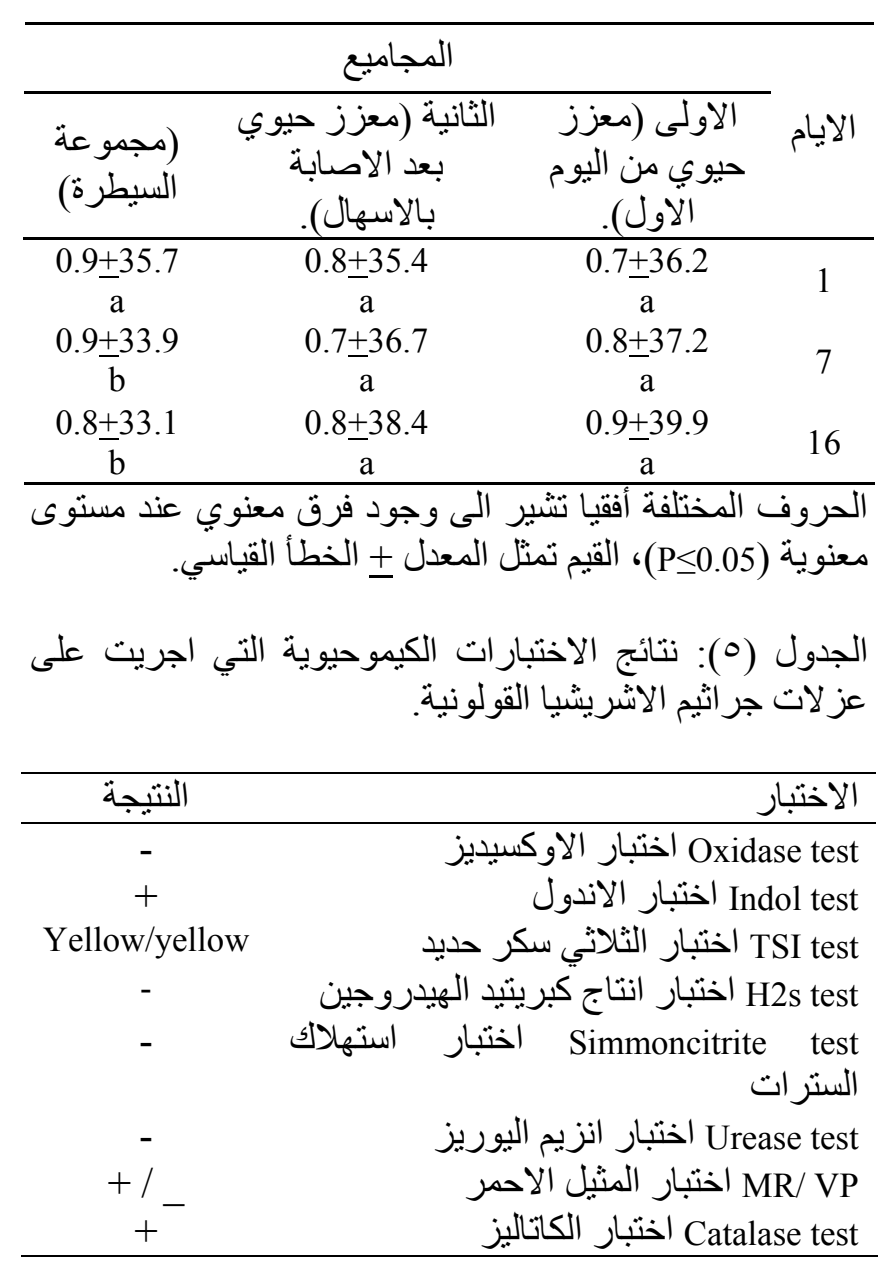

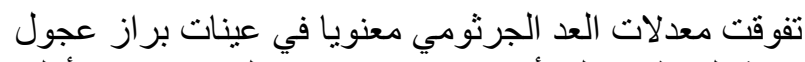

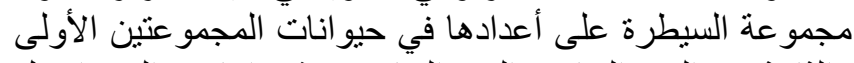

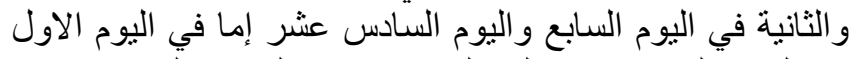

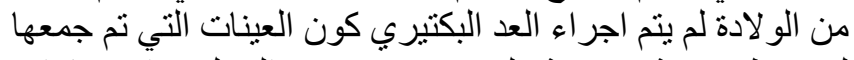

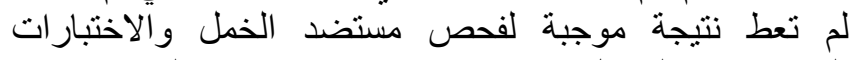

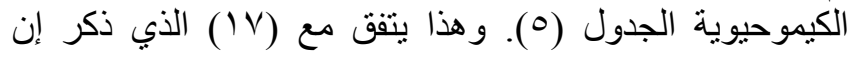




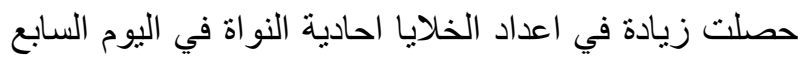

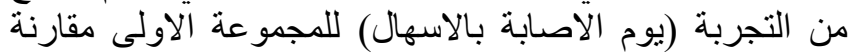

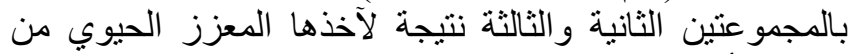

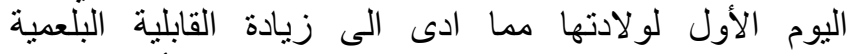

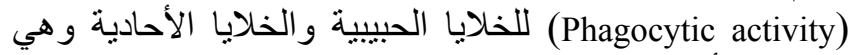

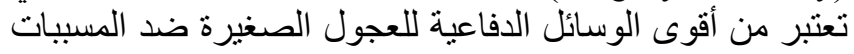

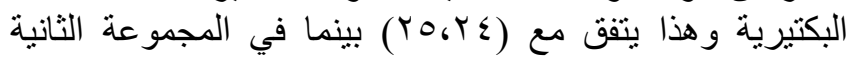

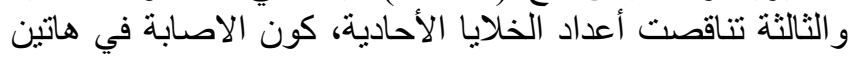

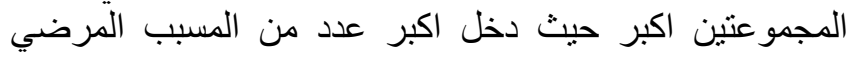

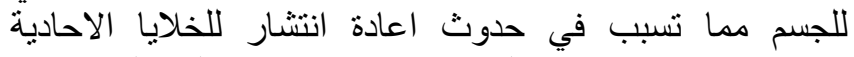

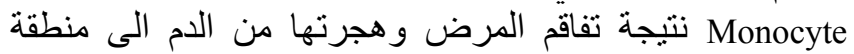

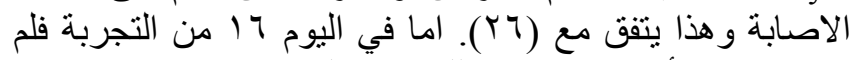

يلاحظ وجود أي فرق معنوي للمجاميع الثناث.

الجدول (9): تأثير اعطاء المعزز الحيوي الامبو في معدلات

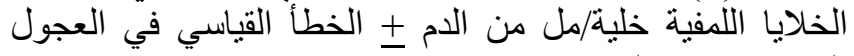

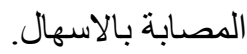

\begin{tabular}{|c|c|c|c|}
\hline \multicolumn{3}{|c|}{ المجاميع } & \multirow[b]{2}{*}{ الايام } \\
\hline السيطرة) & 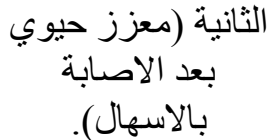 & حيوي من الاولى (معزز & \\
\hline$\underset{\mathrm{a}}{0.94 \pm 3.972}$ & $\begin{array}{c}2.28+3.863 \\
\mathrm{a}\end{array}$ & $2.90 \pm 3.944$ & 1 \\
\hline $3.20+4.900$ & $\begin{array}{c}2.27 \pm 5.145 \\
\mathrm{a}\end{array}$ & $\frac{2.18+4.295}{b}$ & 7 \\
\hline $2.48+4.750$ & $\frac{1.35 \pm 6.668}{\mathrm{a}}$ & $\frac{3.06+7.194}{\mathrm{a}}$ & 16 \\
\hline
\end{tabular}

الحروف المختلفة أفقيا تشير الى وجود فرق معنوي عند مستوى معنوية (P<0.05).

الجدول (•) (1): تأثير اعطاء المعزز الحيوي الامبو في معدلات

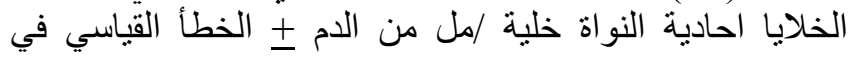
العجول المصابة بالاسهال.

\begin{tabular}{|c|c|c|c|}
\hline \multicolumn{3}{|c|}{ المجاميع } & \multirow[b]{2}{*}{ الايام } \\
\hline (السيطرة عجمة & 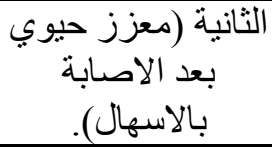 & حيوي من الاوليوم (معزز & \\
\hline $\begin{array}{c}0.40 \pm 0.358 \\
\mathrm{a}\end{array}$ & $\begin{array}{c}0.49 \pm 0.346 \\
\mathrm{a}\end{array}$ & $\begin{array}{c}0.56 \pm 0.361 \\
\mathrm{a}\end{array}$ & 1 \\
\hline $0.30 \pm \frac{ \pm 0.300}{\mathrm{~b}}$ & $\frac{0.58+0.304}{\mathrm{~b}}$ & $\begin{array}{c}0.42 \pm 0.513 \\
\mathrm{a}\end{array}$ & 7 \\
\hline $\begin{array}{c}0.44 \pm 0.325 \\
\mathrm{a}\end{array}$ & $\frac{0.46+0.326}{b}$ & $\begin{array}{c}0.51 \pm 0.334 \\
\mathrm{a}\end{array}$ & 16 \\
\hline
\end{tabular}
معنوية (P<0.05).
الخلايا العدلات كونها الخط الدفاعي الاول في الجسم وجاءت هذة أناء

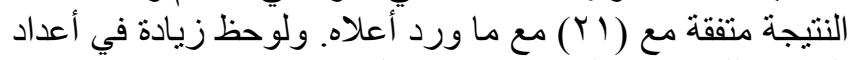

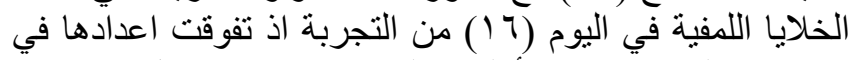

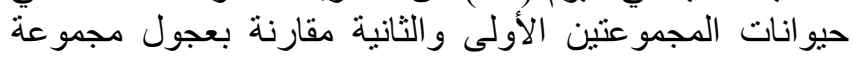

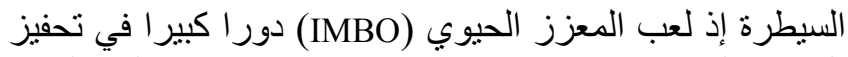

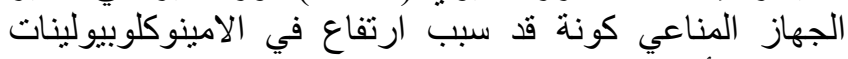
المناعية لأنه يحفز المناعة بنو عيها (الخلوية واعية والخلطية وخاصنة و هذه النتيجة جاءت متفقة ايضا مع (T-CD4, T-CD8)cell -

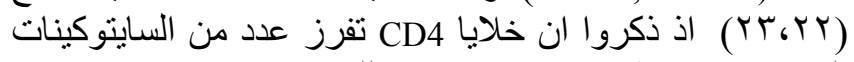

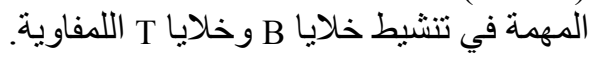

الجدول (V): تأثير اعطاء المعزز الحيوي في معدلات العد الكلي

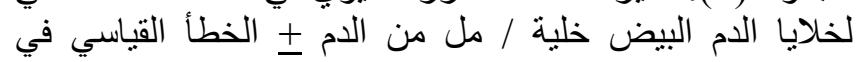

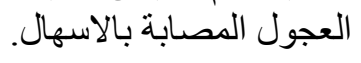

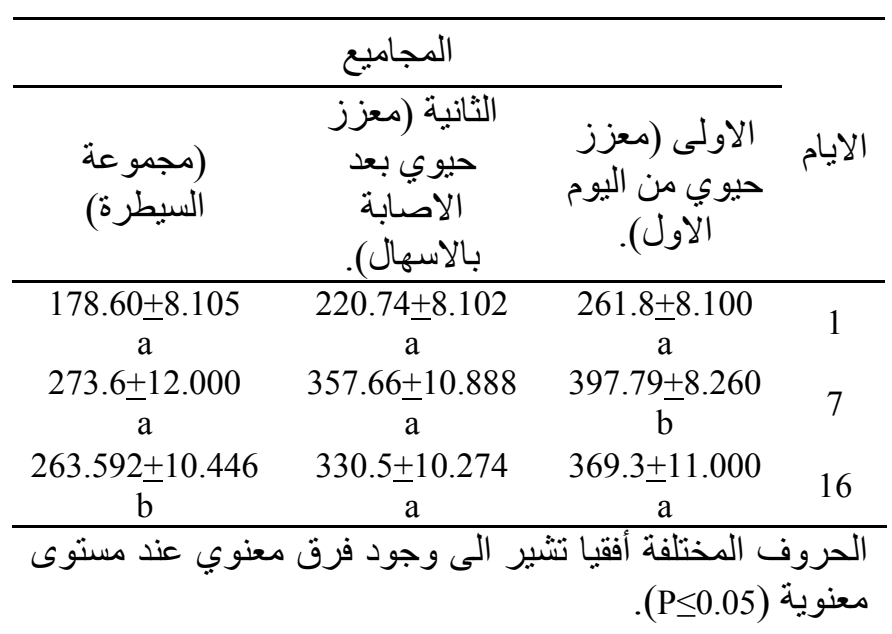

الجدول (^): تأثثير اعطاء المعزز الحيوي الامبو في معدلات

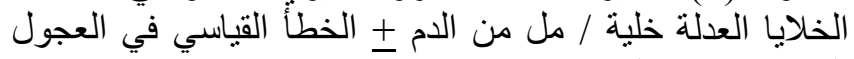

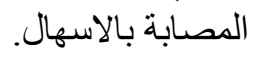

\begin{tabular}{|c|c|c|c|}
\hline \multicolumn{3}{|c|}{ المجاميع } & \multirow[b]{2}{*}{ الايام } \\
\hline (مجمو عة السيطرة) & 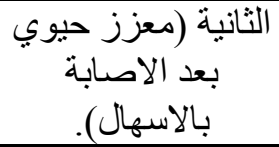 & الاولى (معزز & \\
\hline $\begin{array}{c}1.85 \pm 3.509 \\
\mathrm{a}\end{array}$ & $\underset{\mathrm{a}}{1.24 \pm 3.548}$ & $\begin{array}{c}3.46 \pm 3.499 \\
\mathrm{a}\end{array}$ & 1 \\
\hline$\frac{2.57 \pm 3.364}{\mathrm{a}}$ & $\frac{2.43 \pm 5.743}{a}$ & $\frac{2.43 \pm 3.160}{b}$ & 7 \\
\hline $\begin{array}{c}0.82+5.175 \\
\mathrm{a}\end{array}$ & $\frac{1.80+3.376}{\mathrm{~b}}$ & $\begin{array}{c}1.48+3 . .240 \\
\mathrm{~b}\end{array}$ & 16 \\
\hline & الى وجود فرق & المختلفة أفقيا ت & \\
\hline
\end{tabular}


الجدول (T (1): تأثير اعطاء المعزز الحيوي الامبو في حجم خلايا الدم المرصوصة \% +

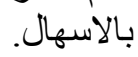

\begin{tabular}{|c|c|c|c|}
\hline \multicolumn{3}{|c|}{ المجاميع } & \multirow[b]{2}{*}{ الايام } \\
\hline (مجيطر عة & الثانية (معزز حيوي & حيوي من الاولى (معزز & \\
\hline $\begin{array}{c}0.5 \pm 36.9 \\
\mathrm{a}\end{array}$ & $\begin{array}{c}0.6 \pm 36.8 \\
a\end{array}$ & $\begin{array}{c}0.8+36.8 \\
\mathrm{a}\end{array}$ & 1 \\
\hline$\underset{\mathrm{a}}{0.9+48.5}$ & $0.8+36.8$ & $0.8+34.5$ & 7 \\
\hline $\begin{array}{c}0.8+55.0 \\
\mathrm{a}\end{array}$ & $\frac{0.8+35.7}{b}$ & $\frac{0.8+35.8}{\mathrm{~b}}$ & 16 \\
\hline
\end{tabular}

الحروف المختلفة أفقيا تشير الى وجود فرق معنوي عند مستوى معنوية (P<0.05).

الجدول (ع 1): تأثير اعطاء المعزز الحيوي الامبو في معدلات البروتين الكلي +الخطأ القياسي في اعلئ العجول المصابة بالأسهال.

\begin{tabular}{|c|c|c|c|}
\hline \multicolumn{3}{|c|}{ المجاميع } & \multirow[b]{2}{*}{ الايام } \\
\hline (مجيطو عة & الثانية (معزز حيوي & حيوي من الاولى (معزز & \\
\hline$\frac{0.2 \pm 6.4}{\mathrm{a}}$ & $\frac{0.3 \pm 6.7}{a}$ & $0.5 \pm 6.8$ & 1 \\
\hline$\frac{0.2 \pm 7.1}{\mathrm{a}}$ & $\frac{0.2 \pm 7.4}{\mathrm{a}}$ & $\frac{0.2 \pm 7.3}{\mathrm{a}}$ & 7 \\
\hline $0.2+7.3$ & $\frac{0.2 \pm 8.9}{\mathrm{a}}$ & $\frac{0.3+8.4}{\mathrm{a}}$ & 16 \\
\hline
\end{tabular}

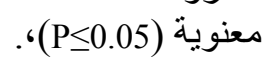

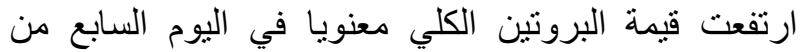

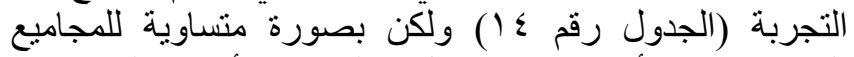

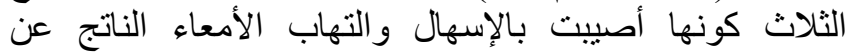

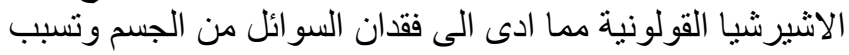

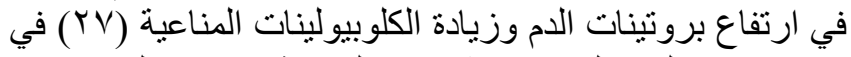

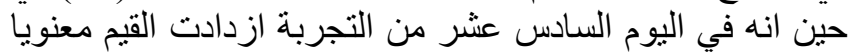

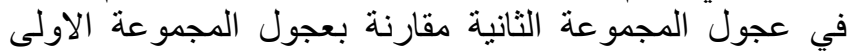

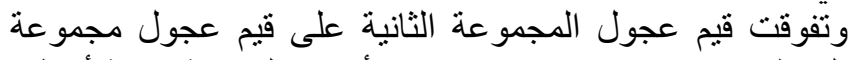
السيطرة و هذا بسبب زيادة مستوى الأجسام المناعية نتيجة العيدة أعطاء

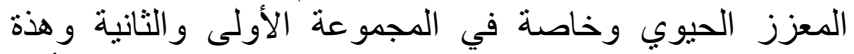

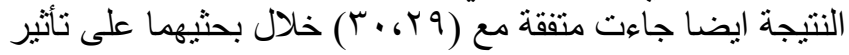

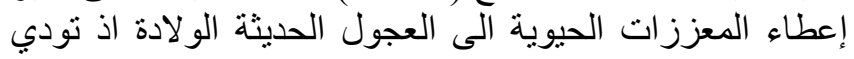

الجدول (11): تأثثر اعطاء المعزز الحيوي الامبو في معدلات

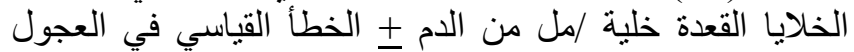

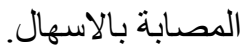

\begin{tabular}{|c|c|c|c|}
\hline \multicolumn{3}{|c|}{ المجاميع } & \multirow[b]{2}{*}{ الايام } \\
\hline (مجمو عة & الثانية (معزز حيوي & حيوي من الاولى (معزز & \\
\hline $0.26 \pm 0.203$ & $0.59 \pm 0.235$ & $0.60 \pm \underset{\mathrm{a}}{0.211}$ & 1 \\
\hline$\frac{0.33 \pm 0.292}{a}$ & $\frac{0.40 \pm 0.273}{a}$ & $\frac{1.30 \pm 0.207}{a}$ & 7 \\
\hline$\frac{0.35 \pm 0.115}{\mathrm{a}}$ & $\frac{0.25 \pm 0.112}{\mathrm{a}}$ & $\frac{0.39 \pm 0.143}{\mathrm{a}}$ & 16 \\
\hline
\end{tabular}

$\frac{a}{a}$ الحروف المختلفة أفقيا تشير الى وجود فرق معنوي عند مستوى (P\05 ) معنوية (P 1 (P.05).

الجدول (T) (M): تأثير اعطاء المعدل الحيوي الامبو معدلات

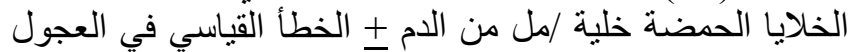

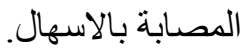

\begin{tabular}{|c|c|c|c|}
\hline \multicolumn{3}{|c|}{ المجاميع } & \multirow[b]{2}{*}{ الايام } \\
\hline (لسيطرة عة & الثانية (معزز حيوي & حيوي من الاولى (معزز & \\
\hline $\begin{array}{c}0.3 \pm 0.081 \\
\mathrm{a}\end{array}$ & $\begin{array}{c}0.56 \pm 0.093 \\
\mathrm{a}\end{array}$ & $\underset{\mathrm{a}}{0.91 \pm 0.097}$ & 1 \\
\hline$\frac{0.16 \pm 0.144}{\mathrm{a}}$ & $\begin{array}{c}0.9 \pm 0.115 \\
\mathrm{a}\end{array}$ & $\begin{array}{c}1.11 \pm 0.092 \\
\mathrm{a}\end{array}$ & 7 \\
\hline$\frac{0.61+0.081}{\mathrm{a}}$ & $\frac{0.67 \pm 0.92}{\mathrm{a}}$ & $\frac{0.21+0.089}{\mathrm{a}}$ & 16 \\
\hline
\end{tabular}

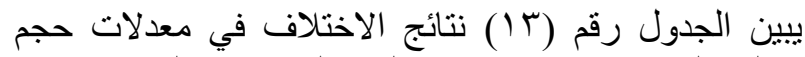

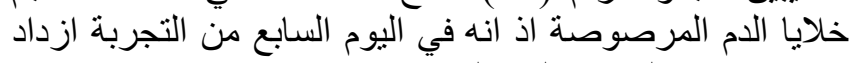

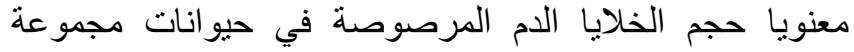

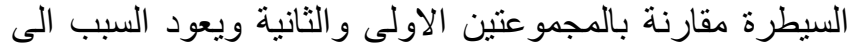

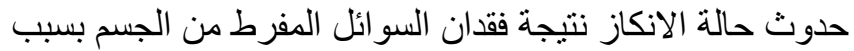

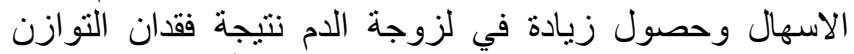

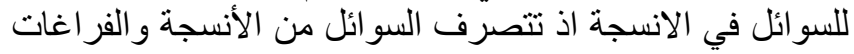

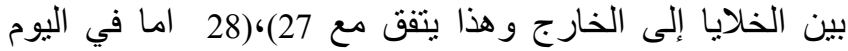

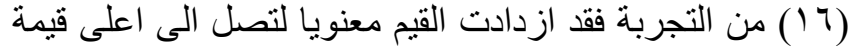

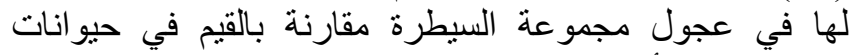
المجمو عتين الأولى و الثانية. 


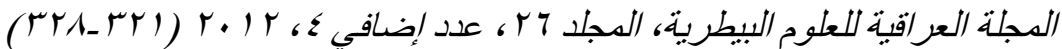

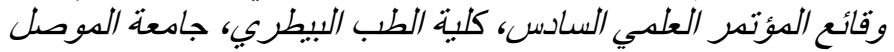

\section{المصادر}

1. 1-Andrews AH, Blowey RC, Boydm H, Eddy RC. Bovine medicine diseases and Husbandary of Cattle. Blackwell Science Ltd. Blackwell Pub Com. 2004;pp:201-204.

2. Timothy HO. Large animal Internal Medicine. $1^{\text {st }}$ ed. 2008., pp:58-72.

3. Fayazi J. Effect of probiotics and antibiotics supplementation in daily milk intake of newborn calves on feed intake, body weight gain and health condition. J Anim Vet Adva. 2010;5:872-875.

4. Jalil B, Gail L, Czarnecki M, Christoph C, Thérèse S, Rachel E, Anderson E, Schiffrin J, Thierry VD. Supplementation of food with Enterococcus faecium (SF68) Stimulates Immune Functions in Young Dogs. J Nutr. 2003;33:1158-1162.

5. Fleige S, Preibinger W, Meywe HHD, Pfaffl MW. Effects of lactuloceon growth performance and intestinal morphology of preruminant calves using a milk replacer containing Enterococcus faecium. Animal. 2007;1:367-373.

6. Coles EH. Veterinary clinical pathology.1998. W.B. Soundrs CO. USA.

7. Feng P, Weagant S. Grant M. Enumeration of Escherichia coli and the coliform bacteria. In: Bacteriological Analytical Manual, 2007. $8^{\text {th }}$ ed. chapter 4 .

8. Miles AA, Misra SS. Laboratory methods in Microbiology. Academic Press. London, England. 1938; pp:38-732.

9 . . جودة محفوظ. التحليل الاحصائي المتقام باستخدام للنشر ,الطبعة الاولى.عمان الاردن.2008.

10. Guthrie A, Land R. Thermoregulation Base mechanisms and hypertheremia. Vet Clinc North Amer Equ Pract.1998;14:45-59.

11. Waller S. Prostoglandins and gastrointestinal tract.Gastroenterology.1978.

12. Kelly WR. Veterinary clinical diagnosis. $2^{\text {nd }}$ ed. Landon, fakenham and reading. 1974.

13. Hossaine SM, Bojarpour M, Mamouei M, Asadian A, Fayazi. Effects of Probiotics and Antibiotic Supplementation in Daily Milk Intake of Newborn Calves on Feed Intake Body Weight Gain, Fecal Scores and Health Condition.2010. Abstract.

14. Al-kinleye SB, Lyayi EA, Afolabi KD. The perform ance, Hematology and cacass trails of broilers as affected by diet supments with or with out Biomin anatural Growth promoter.World J Agric Sci. 2008;(4):467-470.

15. Guerra NP, Bernandez PF, Mendez J, Cachaldora P, Castro, LP. Production of four potentially probiotic lactic acid bacteria and their evaluation as feed additives for weaned piglets. Ani Feed Sci Tech; 2007.134: 89-107.

17

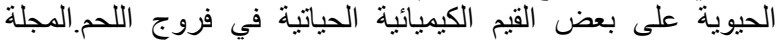

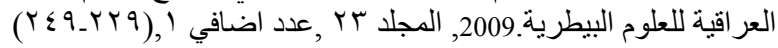

17. Ohya T, Marubashi T, Ito H. Significance of fecal volatile fatty acids in shedding of E. Coli O157 from calves:experimental infection and preliminary use of probiotic product. J Vet Med Sci. 2001;62:11511155.

18. Kramer BW, Moss TJ, Willet KE, Newham JP, SLY PD, Kallapur SG, Ikegami $\mathrm{M}$, Job $\mathrm{AH}$. Dose and time response after intra aminiotic endotoxin in preterm lambs. Am J Respir Crit Care Med. 2001;164(6):982-988.

19. Fleige S, Preibinger W, Meyer HH. The immunomodulatory effect of lactulose on Enterococcus faecium fed preruminant calves.www.jas.fass.org. 2009;87:1731- 1738 .

20. Bai AP, Ouyang Q, Zhang W, Wang CH, SF Li. Probiotics inhibit TNF-alphainducedinterleukin-8 secretion of HT29 cells. World J Gastroenterol. 2010; 10:455-457.

21. Balk RA. Endotoximia in critically ill patients why reliable test could be beneficial. Crit. 2002;6:289-298.

22. Bloom, B, Salgame RP, Diamond B. Revisiting and revising suppressor T-cells. Immunol. Today.1992;13:131-136
الى زيادة الكلوبيولين المناعي نوع IgG وبعض القيم الدموية

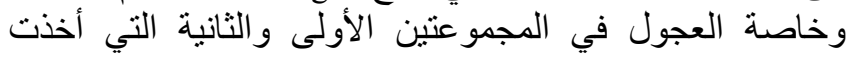

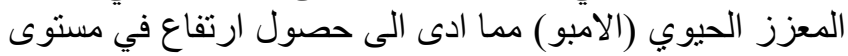

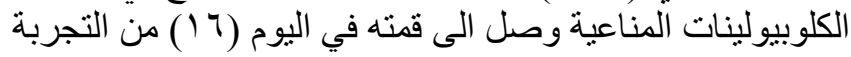

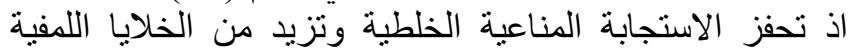

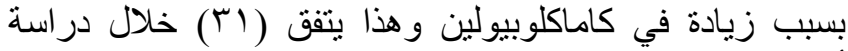

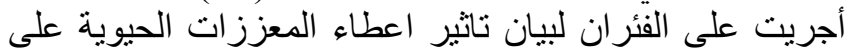

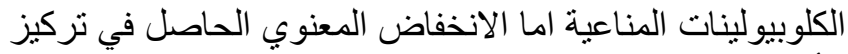

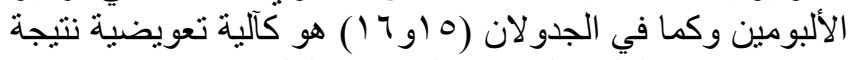

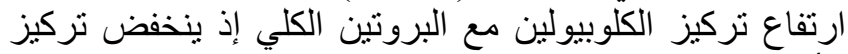

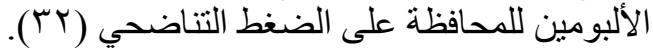

الجدول (10): تأثثير اعطاء المعزز الحيوي الامبو في معدلات

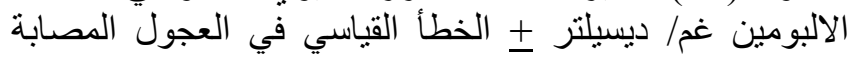

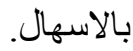

\begin{tabular}{|c|c|c|c|}
\hline \multicolumn{3}{|c|}{ المجاميع } & \multirow[b]{2}{*}{ الايام } \\
\hline السيطرة & 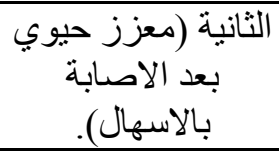 & حيوي من اليوم (معزز & \\
\hline $0.3 \pm 2.9$ & $\begin{array}{c}0.2 \pm 2.6 \\
\mathrm{a}\end{array}$ & $\begin{array}{c}0.3 \pm 2 . .6 \\
\mathrm{a}\end{array}$ & 1 \\
\hline$\frac{0.5 \pm 3.1}{\mathrm{a}}$ & $0.4 \pm 2.7$ & $\frac{0.5 \pm 2.3}{\mathrm{a}}$ & 7 \\
\hline$\frac{0.1 \pm 3.6}{\mathrm{a}}$ & $\frac{0.2 \pm 2.8}{\mathrm{~b}}$ & $0.2+2.1$ & 16 \\
\hline
\end{tabular}

الجدول (7 (1): تأثير اعطاء المعزز الحيوي الامبو في معدلات

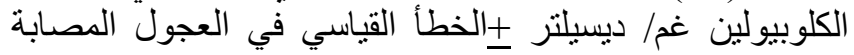

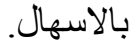

\begin{tabular}{|c|c|c|c|}
\hline \multicolumn{3}{|c|}{ المجاميع } & \multirow[b]{2}{*}{ الايام } \\
\hline (مجيطو عة & الثانية (معزز حيوي & حيوي من الاولى (معزز & \\
\hline $0.2 \pm 4.2$ & $0.21 \pm 4.6$ & $0.11 \pm 4.0$ & 1 \\
\hline$\overline{\mathrm{a}}$ & $\bar{a}$ & $\bar{a}$ & 1 \\
\hline $0.2 \pm 4.4$ & $0.2 \pm 4.6$ & $0.2 \pm 4.5$ & 7 \\
\hline $\mathrm{a}$ & a & a & \\
\hline $0.2+4.0$ & $0.2 \pm 5.6$ & $0.2 \pm 5.2$ & 16 \\
\hline $\mathrm{b}$ & $\mathrm{a}$ & $\mathrm{a}$ & \\
\hline
\end{tabular}




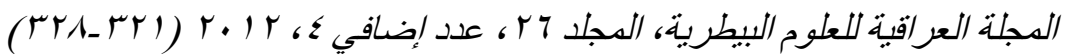

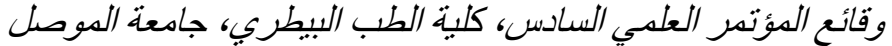

28. AL-Saidy MY. Effect of Probiotic Bacteria on Immunoglobulin G concentration and Other Blood Components of Newborn Calves. J Anim Vet Advan. 2010; 9:3: 604-609.

29. Schalm, OJ, Camol E. Veterinary hematology.philadelphia. Le and Febiger. 2000.

30. Abas I, Kutwy HC, Kagraman R, TOker NY, Ozcelik D. Ates F. Kacaki A. Effect of organic acid and bacterial direct fed microbial on fattening performance of kivircik male yearling lamb. $P$ G.2007;(2):149-154.

31. Sarinee, k.; Tnongong, T.B.and chavahanikual, V. Blood hematological cholesterol profile and antibody titer response of broiler with added probiotics containing both bacteria and yeast an or antibiotics in drinking water. Thai J Vet Med. 2008;38(4):45-67.

32. Latimar KS, Manhaffey EA, Prasses KW. Clinical pathology. $4^{\text {th }}$ ed. Iowa State Press. 2003;pp:162-175.
23. Erickson KL, Hubbard NE. Probiotic immunomodulation in health and disease, J Nutr. 2000;130: $403-409$.

24. Shin-Ichi K, Tomoya Y, Naoto N, Yimin C. Feeding of Lactic Acid Bacteria and Yeast on Growth and Diarrhea of Holstein Calves. J Anim Vet Adva. 2010; 9: 1112-1114.

25. Kantari, C, Pederzoli-Ribeil M, Witko-Sarsat V. The role of neutrophils and monocytes in innate immunity. Contrib Microbiol. 2008; 15:118-146.

26. Dekker PEP, HoveT, Velde AA, Daventer SJ, Poll T. UP regulation of monocyte urokinase plasminogen activator receptor human endotoxine infection and immunity. 2000;68(4):2156-2160.

27. Radostits, OMO, Blood DC, Gay CC, Hinchlff KW. Veterinary medicine a text book of the disease of cattle, sheep, pigs and goats.10th ed. Elsevir Sounder Spain Company.2007. 